\title{
A UN SIGLO DE LA LEY SÁENZ PEÑA: \\ EN BUSCA DE UN EQUIVALENTE \\ CONTEMPORÁNEO
}

OSVALDO IAZZETTA ${ }^{1}$

\section{La democracia}

\section{en el Primer Centenario}

La Argentina del Primer Centenario expresaba una promesa de expansión económica y movilidad social que explica la fuerte atracción que estas tierras ejercieron para millones de inmigrantes provenientes de otras latitudes. Ese optimismo, sin embargo, resultaba contrariado por el profundo malestar que provenía de la escena política. Basta revisar el tono crítico del balance que Joaquín V. González (1979) trazó en ese aniversario al lamentar que el sufragio aún constituyera una aspiración incumplida.

El voto, herramienta democrática por excelencia, era objeto de sospecha, desvirtuado por la manipulación de padrones y viciado por prácticas fraudulentas que desnaturalizaban su sentido.

La Ley Sáenz Peña -que dos años después del Centenario ofreció respuesta a ese malestar- permitió emprender, con la elección de Hipólito Yrigoyen (1916), la ardua marcha hacia una democracia con "participación ampliada» que no cesaría de expandirse, en especial tras el reconocimiento del voto femenino y la inclusión de nuevos actores sociales.

Esta ley creó las condiciones para encarar una modernización de la vida política más consonante con los logros que el país mostraba en materia económica, social y cultural. Por ende, no resulta exagerado sostener que las reglas de juego impuestas por esta ley sentaron las bases de nuestra democracia moderna.

Sin embargo, como aclara Hilda Sabato (2005: 27) «...esta pieza legislativa fue fundamental no porque innovara en cuanto a la amplitud del derecho a voto sino porque introdujo modificaciones en otras dimensiones que eran decisivas. Desde el punto de vista del sufragio, dos fueron clave: la obligatoriedad y el secreto del voto».

En primer lugar, esta ley convirtió en un deber lo que hasta entonces era sólo un derecho escasamente ejercido por los

\footnotetext{
${ }^{1}$ Osvaldo lazzetta es Profesor de la Facultad de Ciencia Política y Relaciones Internacionales de la Universidad Nacional de Rosario e Investigador del Consejo de Investigaciones de esa Universidad.
} 
ciudadanos habilitados para ello. Sus impulsores se propusieron rehacer sobre nuevas bases el sistema político argentino introduciendo un elemento coactivo que, paradojalmente, constituyera a los habitantes en ciudadanos ${ }^{2}$.

Aunque en el siglo XIX ya existía un reconocimiento del derecho al voto más amplio del que se supone (Sabato 1998), la participación electoral efectiva era limitada y sólo una muy baja proporción de la población ejercía sus derechos electorales ${ }^{3}$.

\section{Los derroteros de la ciudadanía política en nuestra experiencia}

Si la conquista del voto es entendida por la literatura clásica como un aspecto crucial en la adquisición de ciudadanía política ${ }^{4}$, la afirmación de ese derecho recién fue posible entre nosotros -aunque de manera parcial al limitarse a los hombres-, cuando se convierte en un deber rodeado de las garantías que le aportaba su carácter secreto.

La coacción a la que se prestaba el voto público y la falta de transparencia en los aspectos procedimentales (manipulación de padrones y del escrutinio) viciaban al acto electoral actuando como un poderoso factor de desaliento. Algunos testimonios como el de Juan Álvarez (1976: 129130), destacan que el fraude y la violencia obstaculizaban el ejercicio del sufragio libre y produjeron «una apatía del cuerpo electoral» pese a las leyes vigentes que, ya antes de 1912, reconocían ese derecho.

Por tal razón, detrás de su aparente sencillez, la Ley Sáenz Peña encerraba una acertada ingeniería institucional que se tornó decisiva para ofrecer respuesta a los problemas que constituían la principal fuente de malestar político en la sociedad argentina de comienzos del siglo XX.

Que ella no haya bastado para asegurar la continuidad de nuestra democracia -como lo prueban las reiteradas interrupciones que se sucedieron luego de 1930 no puede atribuirse a esta ley ni desmerece su aporte en nuestra traumática construcción democrática. Como en otros temas de nuestro pasado, sólo podremos hallar respuesta interrogándonos sobre el uso y apropiación que de ella hicieron los actores políticos durante el tumultuoso medio siglo que transcurrió entre 1930 y 1983.

Tal vez no haya sido ajeno a ello el desprecio que las clases dirigentes argentinas

\footnotetext{
${ }^{2}$ Véase sobre este punto Gutiérrez y Romero (1995: 156).

${ }^{3}$ Ello no debe atribuirse únicamente a las restricciones del diseño institucional vigente o a las prácticas fraudulentas que desalentaron su ejercicio, sino también a la disponibilidad de otros canales de expresión, participación y movilización política que la ciudadanía percibía como no menos atrayentes y efectivos que el ejercicio del voto. Véase al respecto Sabato (1998; 2005) y Gutiérrez y Romero (1995).

${ }^{4}$ Véase Marshall (1998).
} 
mostraron por los procesos electorales, negándoles legitimidad como medio para desplazar a sus gobernantes, lo que contribuyó a aceptar la idea de que cuando las elecciones no los favorecen los derrotados no están obligados a reconocer los resultados 5 .

De todos modos, la discontinuidad de la democracia y la suspensión de derechos y garantías que los regímenes autoritarios impusieron a su paso, alteró la posibilidad de confirmar la secuencia lineal y acumulativa de los derechos ciudadanos que Marshall (1998) describió basado en la experiencia inglesa ${ }^{6}$.

Aunque esa expectativa carece de sustento histórico -es ilusorio imaginar una vía única en la construcción de la ciudadanía y la democracia- ese trayecto singular no dejó de tener implicancias en el tipo de ciudadanía efectivamente conquistada, privándola de la trama institucional y soportes que da por existentes la teoría democrática contemporánea ${ }^{7}$.
En nuestra experiencia, esa discontinuidad significó que los derechos ciudadanos no hayan cobrado forma por acumulación sino por exclusión, de modo tal que la conquista de unos derechos en ocasiones vino acompañada de la supresión completa o parcial de otros ${ }^{8}$.

Incluso la recuperación de derechos políticos que fue posible a partir de 1983, coexiste tensamente con una desmejora en materia de derechos sociales ya disponibles y con derechos civiles que, aunque proclamados formalmente, no logran garantizarse con el mismo nivel de homogeneidad social y territorial que los políticos.

Este desequilibrio ha sido adecuadamente captado por los informes del PNUD (2004; 2008) que destacan como uno de los principales logros de la región la existencia de una democracia electoral sin que ello equivalga por el momento, a una democracia de ciudadanía.

\footnotetext{
${ }^{5}$ Esta es una interpretación que ensaya Dahl (1989: 127-129) en su consagrado libro La poliarquía. Participación y oposición. En ese libro, Dahl destina un apartado al caso argentino indagando las causas del fracaso de nuestra democracia. Este autor centra su explicación en la falta de creencia en la legitimidad de las elecciones y en la exclusión de los inmigrantes de los procesos electorales cuando estos representaban -según el censo demográfico de 1914- la mitad de los ciudadanos en Buenos Aires.

${ }^{6}$ Esa distancia también es señalada entre los mismos países europeos occidentales, tal como destaca Déloye (2004). Sobre la singularidad de nuestra región en este tema puede consultarse entre otros textos, a Murilo de Carvalho (1995) y Sabato (1999).

7 O'Donnell (2001) ha alertado sobre los riesgos que conlleva la trasposición mecánica de las secuencias registradas en los países noratlánticos, recordando que en estas experiencias la extensión de los derechos políticos estuvo precedida por la existencia de una densa trama de derechos civiles y subjetivos que no se verificó entre nosotros.

${ }^{8}$ Véase Botana (2004: 33).
} 


\section{Las elecciones, un siglo después \\ de la Ley Sáenz Peña}

La grata novedad que introdujo el ciclo democrático abierto hace tres décadas radica en que nuestro problema ya no proviene-como en otros tiempos-del momento electoral. Las elecciones libres, periódicas y competitivas son un pilar decisivo de las democracias y ese componente parece hoy más asegurado que en otras etapas de nuestra historia política ${ }^{9}$. Asimismo, el compromiso expresado por la ciudadanía en ese instante crucial de la vida democrática revela la fuerte implantación del voto como recurso democrático básico y como sustento del poder legítimo ${ }^{10}$.

Este no es un dato menor, pues en una democracia que aún acusa signos de debilidad institucional, las elecciones son una de las pocas instituciones que efectivamente han funcionado en esta etapa histórica ${ }^{11}$.

Ahora bien, celebrar este logro no significa ignorar ciertos aspectos que aún ensombrecen esa conquista. Dentro de ellos destacamos preferentemente, los contextos que rodean a las elecciones como así también, lo que sucede después de éstas.

\section{a. Sobre los contextos}

Son bienvenidas las elecciones, y especialmente su periodicidad y perdurabilidad en el tiempo. Sin embargo, evaluar en qué contextos se registran nuestras elecciones nos brinda la posibilidad de dimensionar cuán necesario resulta expandir la democratización más allá del momento electoral.

Como es sabido, Schumpeter es uno de los autores clásicos que señaló a la competencia electoral como el procedimiento democrático por excelencia. En su libro Capitalismo, Socialismo y Democracia -publicado en 1942- estableció ciertas condiciones para el éxito del «método democrático» advirtiendo que si un mismo mecanismo o procedimiento (en este caso las elecciones) funciona de manera diferente en épocas distintas y en lugares distintos, eso indica que su funcionamiento depende de condiciones extrañas al mismo ${ }^{12}$. No es preciso compartir todas las exigencias enumeradas por Schumpeter para aceptar que el funcionamiento del mecanismo electoral efectivamente varía según las condiciones externas que lo rodean.

\footnotetext{
9 Desde 1983 se han celebrado en nuestro país 7 elecciones presidenciales, 8 rondas de elecciones de gobernadores y 15 legislativas.

10 Véase Cheresky (2006a: 15-16).

${ }^{11}$ Véase Levitsky y Murillo (2007) y O’Donnell (1996).

12 Véase Schumpeter (1996: 368).
} 
Aunque las elecciones encierran un enorme potencial democrático y constituyen una palanca decisiva para avanzar hacia la conquista de nuevos derechos, en aquellos contextos en los que el oficialismo controla cuantiosos recursos, en los que gobierno, partido gobernante y Estado permanecen confundidos y la autonomía de las organizaciones de la sociedad civil se ve comprometida, las elecciones tienden a reproducir ese desbalance de fuerzas, aun cuando las reglas de juego sean iguales para todos los jugadores que compiten.

Es preciso no permanecer indiferentes ante estos signos pues ellos no sólo ensombrecen el momento electoral sino también ponen en evidencia los límites de la democracia para promover una desconcentración del poder, más allá de ese instante fugaz.

Las elecciones reflejan la distribución cuantitativa de las preferencias ciudadanas, sin embargo, cuando éstas vienen acompańadas de una persistencia de los oficialismos o de un reeleccionismo indefinido (como en Formosa, donde el actual gobernador ejerce su quinto mandato) revelan algo más que la simple distribución de preferencias electorales.

Detrás de ellas se ocultan recursos dispares y desequilibrios en las oportunidades de acceso a los cargos electivos, y en esas condiciones las elecciones actúan como un medio muy limitado de democratización resultando condenadas a reproducir las inclinaciones de ese contexto.

Conviene recordar que en el nivel subnacional un tercio de los 24 distritos electorales del país aún no ha registrado ninguna experiencia de alternancia y se mantiene gobernado por el mismo partido o coalición desde 1983. Este predominio genera una asimetría de recursos entre oficialismo y oposición y convierte al partido gobernante en una agencia semiestatal ${ }^{13}$, configurando un escenario poco propicio para tornar posible su rotación. Valorar las elecciones libres y periódicas no debe convertirse en complacencia frente a la concentración de poder que las rodean y que se manifiesta preferentemente -aunque no exclusivamente- en el reeleccionismo indefinido y en las ventajas a las que tienen acceso los oficialismos, convirtiendo el control del Estado en una fuente de desequilibrios que bloquea toda posibilidad de alternancia.

Esta revisión se ha apoyado en ciertos rasgos del nivel subnacional que se ubican al límite y tal vez no sean representativos de aquellos distritos en los que la alternancia y la competencia electoral parecen aseguradas. A nadie escapa, sin embargo, que lo que sucede en aquellos distritos no

13 Tomamos la expresión de Cheresky (2006b: 13). 
queda confinado a sus límites sino que se proyecta en el nivel nacional, trasladando sus prácticas a instituciones nacionales en las que tienen representación.

\section{b. Los procedimientos no se limitan al acceso al gobierno sino también a su ejercicio}

Los procedimientos de una democracia no definen sólo un modo de acceso al gobierno sino también un modo de ejercerlo ${ }^{14}$, y esto último nos revela cómo desempeñan sus cargos las autoridades una vez electas.

Si nos centramos en este eje, debemos recordar que la dinámica de la democracia instaurada en 1983 estuvo dominada por crisis económicas muy severas (1989 y 2001), que pusieron a prueba su estabilidad y crearon situaciones de excepción favoreciendo la irrupción de liderazgos fuertes y la concentración de recursos de gobierno en el Poder Ejecutivo ${ }^{15}$.

El sentido de urgencia gestado bajo dichas crisis mantuvo fuerte interacción con un modo de entender el ejercicio del poder que, sacando provecho de aquella excepcionalidad, pugna por ampliar la autono- mía decisoria del Ejecutivo en desmedro de otros poderes y órganos de control estatal. Ello se tradujo en una ecuación que, según O’Donnell, se distingue por maximizar los mecanismos estatales que permiten la concentración y efectividad de las decisiones y por minimizar aquellos relativos a la transparencia y rendición de cuentas ${ }^{16}$.

Esta manera de entender el ejercicio del poder encierra una idea de la democracia como un puro sistema de mayoría basado en elecciones. Sin embargo, el principio mayoritario no basta para fundar las instituciones democráticas y las elecciones no garantizan que un poder sea plenamente democrático; para que ello resulte posible debe estar sometido a pruebas de control y validación que sean concurrentes y complementarias de la expresión mayoritaria ${ }^{17}$. En palabras de Rouquié (2011: 192), «las elecciones no hacen por sí solas las democracias, también se necesita el Estado de derecho y el ejercicio reglamentado del poder».

Contar con elecciones periódicas y competitivas es una conquista que no podemos minimizar, más aún, en países en los

\footnotetext{
${ }^{14}$ Acerca de esta distinción puede consultarse Mazzuca (2002; 2006).

${ }^{15}$ Véase al respecto el tratamiento que Quiroga (2005) efectúa sobre las situaciones de emergencia que se han reiterado en este ciclo democrático.

${ }^{16}$ Véase la entrevista efectuada por José Natanson, «Sobre los tipos y calidades de democracia», Página 12, 27/02/2006. También puede consultarse el concepto de democracia delegativa que O'Donnell (1997) acuñó para retratar situaciones de este tipo en nuestra región.

17 Véase Pierre Rosanvallon «Reinventar la democracia es hacerla más responsable», Clarín, 04/05/2009.
} 
que esa posibilidad ha sido excepcional y esporádica. Sin embargo, así como debemos permanecer atentos a los contextos que las rodean también debemos interesarnos por lo que ocurre después de aquéllas. Eso implica, por consiguiente, hacer foco sobre cómo se ejerce el poder y cómo se toman decisiones colectivas vinculantes, una vez electas nuestras autoridades.

\section{Breves palabras finales}

Si la Ley Sáenz Peña fue una respuesta adecuada a los problemas políticos que eran motivo de malestar a comienzos del siglo $X X$, resulta imperioso reunir $y$ convocar una lucidez y voluntad política equivalente, para enfrentar los problemas que expresan otras formas de desencanto y frustración en este nuevo siglo.

La democracia no suprime las disparidades de poder, sólo representa una de las formas posibles de organizar políticamente esas relaciones, distinguiéndose de otras variantes por crear e institucionalizar «el poder desde abajo», el poder de los gobernados ${ }^{18}$. Para ello el ciudadano dispone de derechos respaldados estatalmente, dentro de los cuales el derecho al voto es una de sus herramientas principales, aunque no la única por cierto.

El potencial igualitario que encierra el momento electoral debe expandirse a otros ámbitos ${ }^{19}$. La vigencia de este principio igualitario que se renueva en cada acto electoral, nos recuerda todo lo que hemos avanzado en estos años de democracia pero, sin dejar de reconocer este logro, es preciso no caer en la auto-complacencia y asumir la democratización como un proceso expansivo ${ }^{20}$ que no se agota en ese instante, por crucial que resulte.

La teoría democrática predominante se ha concentrado en el modo de acceso a los cargos públicos soslayando otras dimensiones, no menos decisivas para una democracia. Los dos aspectos destacados en este breve balance -los contextos que rodean las elecciones y el modo en que se ejerce el poder- señalan desafíos que imponen expandir ese impulso democratizador más allá del acto electoral.

Si fuera posible condensar una agenda capaz de guiarnos en los próximos cien años, tal como la Ley Sáenz Peña lo hizo un siglo atrás, no vacilaría en señalar aquellas tareas que hicieran posible una mayor desconcentración y dispersión del

18 Seguimos en este a aspecto a Vargas Cullell (2011: 72).

${ }^{19}$ Según O'Donnell (2010: 287-291), las elecciones son por excelencia, el único tipo de actividad política para el que hay un criterio de igualdad entre los que participan y si bien son un momento pasajero, ellas operan como oportunidades institucionalizadas para nuevas reapropiaciones, potencialmente movilizantes, del verdadero significado de la autoridad y los poderes de Estados y gobiernos.

${ }^{20}$ Ackerman (2006: 150) sugiere pensar las tareas democráticas como expansivas y no como terminales. 
poder $^{21}$. El siglo transcurrido nos enseñó a valorar nuestro derecho al voto, vedado durante los regímenes autoritarios y limitado por proscripciones en los ensayos democráticos que alternaron con aquéllos. Afortunadamente, ese derecho ha queda- do asegurado en las tres últimas décadas pero, una vez conquistado ese umbral, son otros los horizontes y otras las tareas que habrán de darle nuevo contenido a la democratización por venir.

\section{Bibliografía}

ACKERMAN, JOHN M. (2006): «Democratización: pasado, presente y futuro», Perfiles Latinoamericanos, № 28, DF-México, FLACSO, julio-diciembre.

ÁLVAREZ, JUAN (1976): Las guerras civiles argentinas, Buenos Aires, Eudeba.

BOTANA, NATALIO (2004): «Dimensiones históricas de las transiciones a las democracias en América Latina», en: La democracia en América Latina. Hacia una democracia de ciudadanas y ciudadanos. Contribuciones para el debate, Buenos Aires, PNUD.

CHERESKY, ISIDORO (2006a): «Elecciones en América Latina: poder presidencial y liderazgo político bajo la presión de la movilización de la opinión pública y la ciudadanía», Nueva Sociedad, No 206, Buenos Aires, noviembre-diciembre.

CHERESKY, ISIDORO (2006b): «La política después de los partidos», en: Isidoro Cheresky (comp.), La política después de los partidos, Buenos Aires, Prometeo.
DAHL, ROBERT (1989): La poliarquía. Participación y oposición, Buenos Aires, Rei. DÉLOYE, YVES (2004): Sociología histórica de 10 político, Santiago de Chile, Lom Ediciones.

GONZÁLEZ, JOAQUín V. (1979): El juicio del sig/o, Buenos Aires, Centro Editor de América Latina. GUTIÉRREZ, LEANDRO y ROMERO, LUIS ALBERTO (1995): Sectores populares, cultura y política. Buenos Aires en la entreguerra, Buenos Aires, Sudamericana.

LEVITSKY, STEVEN y MURILLO, MARÍA V. (2007): «Teorías sobre instituciones débiles: lecciones del caso argentino», en: Sergio Emiliozzi, Mario Pecheny y Martín Unzué (comps.), La dinámica de la democracia. Representación, instituciones y ciudadanía en Argentina, Buenos Aires, Prometeo. MAZZUCA, SEBASTIÁN (2002): «¿Democratización o burocratización? Inestabilidad del acceso al poder y estabilidad del ejercicio del poder en América Latina», Aracauria 7.

${ }^{21}$ Para ello, la democratización deberá ser entendida como un proceso abierto «... por medio del cual la organización democrática de relaciones de poder se disemina en una sociedad" y atraviesa los distintos ámbitos de la vida social (Vargas Cullell 2011: 71-72). Sobre el mismo tema también puede consultarse Whitehead (2011). 
MAZZUCA, SEBASTIÁN (2006): «Ampliando las concepciones sobre la democracia: acceso al poder versus ejercicio de poder», en: Víctor Alarcón Olguín (coord.), Metodologías para el análisis político. Enfoques, procesos e instituciones, México, Universidad Autónoma Metropolitana, Sede Iztapalapa.

MARSHALL, T. H. (1998): «Ciudadanía y clase social», en: T. H. Marshall y T. Bottomore, Ciudadanía y clase social, Madrid, Alianza Editorial.

MURILO de CARVALHO, JOSÉ (1995): Desenvo/vimiento de la ciudadanía en Brasil, México, El Colegio de México, Fondo de Cultura Económica.

O'DONNELL, GUILLERMO (1996): «Otra institucionalización», Ágora. Cuadernos de Estudios Políticos, $N^{\circ} 5$, Buenos Aires, invierno.

O'DONNELL, GUILLERMO (1997): ¿Democracia delegativa?, en: Guillermo O'Donnell, Contrapuntos. Ensayos escogidos sobre autoritarismo y democratización. Buenos Aires, Paidós.

O'DONNELL, GUILLERMO (2001): «Régimen y Estado en la teoría democrática», Temas y Debates, $\mathrm{N}^{\circ}$ 4-5, Rosario, julio.

O'DONNELL, GUILLERMO (2010): Democracia, agencia y Estado. Teoría con intención comparativa, Buenos Aires, Prometeo.

PROGRAMA DE NACIONES UNIDAS PARA EL DESARROLLO (PNUD) (2004): La democracia en América Latina. Hacia una democracia de ciudadanas y ciudadanos, Buenos Aires.
PROGRAMA DE NACIONES UNIDAS PARA EL DESARROLLO (PNUD) (2008): Democracia/Estado/Ciudadanía. Hacia un Estado de y para la Democracia en América Latina, Lima, PNUD.

QUIROGA, HUGO (2005): La Argentina en emergencia permanente, Buenos Aires, Edhasa.

ROUQUIÉ, ALAIN (2011): A la sombra de las dictaduras. La democracia en América Latina, Buenos Aires, Fondo de Cultura Económica.

SABATO, HILDA (1998): La política en las calles. Entre el voto y la movilización. Buenos Aires, 1862-1889, Buenos Aires, Sudamericana.

SABAT0, HILDA (2005): Pueblo y política. La construcción de la República, Colección Claves para Todos No 40, Buenos Aires, Capital Intelectual. SABATO, HILDA (coord.) (1999): Ciudadanía política y formación de las naciones. Perspectivas históricas de América Latina, México, El Colegio de México, Fondo de Cultura Económica.

SCHUMPETER, JOSEPH A. (1996): Capitalismo, Socialismo y Democracia, Barcelona, Folio, t. 2.

VARGAS CULLELL, JORGE (2011): "La calidad de la democracia y el estudio comparado de la democratización», en: Revista Latinoamericana de Política Comparada, $N^{\circ} 5$, Centro Latinoamericano de Estudios Políticos. Quito, Julio.

WHITEHEAD, LAURENCE (2011): Democratización.

Teoría y experiencia, México, Fondo de Cultura Económica.

\section{Registro bibliográfico}

IAZZETTA, OSVALDO

«A un siglo de la Ley Sáenz Peña: en busca de un equivalente contemporáneo», en: ESTUDIOS SOCIALES, revista universitaria semestral, año XXII, No 43, Santa Fe, Argentina, Universidad Nacional del Litoral, segundo semestre, 2012, pp. 279-287. 
Toda correspondencia debe dirigirse a:

ESTUDIOS SOCIALES

revista universitaria semestral.

Casilla de Correo 353,

Correo Argentino sucursal Santa Fe,

(3000) Santa Fe, Argentina.

Secretaría de Redacción:

e-mail: estudiossocialesunl@gmail.com

\section{Los trabajos con pedido de publicación}

deben ser inéditos y observar en su presentación

las siguientes recomendaciones:

1 Deben enviarse una copia impresa y una en formato electrónico, esta última a la siguiente dirección: estudiossocialesunl@gmail.com.

2 Con respecto a la extensión de los trabajos debe considerarse como límite máximo: 75.000 caracteres para ARTÍCULOS; 30.000 para COMUNICACIONES, NOTAS Y COMENTARIOS; y 10.000 para NOTAS BIBLIOGRÁFICAS.

3 Los trabajos deben ir acompañados de: a) un resumen de no más de 150 palabras, en castellano y en inglés; b) los principales descriptores o palabras claves, no más de cinco; c) las referencias institucionales del autor, con la dirección postal, teléfono y e-mail.

4 Los cuadros y gráficos se incluirán en hojas separadas del texto, numerados y titulados correctamente. Los gráficos deben presentarse confeccionados en láser para su impresión directa.

5 Las NOTAS al pie de página deben enumerarse correlativamente observando el siguiente orden: a)
Nombres y apellido del autor (en minúscula); b) título de la obra (destacado en cursiva); en el caso de artículos, éste irá entre comillas destacándose la obra o revista que lo incluye; c) vol., $\mathrm{N}^{\circ}$, etc.; d) lugar de edición; e) editorial; f) fecha de la publicación; g) p. o pp., si correspondiera.

6 Si se detalla BIBLIOGRAFíA, ésta se incluirá al final del trabajo, ordenada alfabéticamente por autor y observando el siguiente orden: a) Apellido y nombres del autor; b) fecha de edición de la obra (entre paréntesis); y los datos del trabajo atendiendo las indicaciones señaladas en el ítem anterior.

7 Para la aprobación de su publicación los trabajos serán sometidos a la consideración del Consejo Editorial y de evaluadores externos.

8 ESTUDIOS SOCIALES no se hace responsable por los trabajos no publicados ni se obliga a mantener correspondencia con los autores sobre las decisiones de selección. 